\section{Comparação de três critérios de classificação de sobrepeso e obesidade entre pré- escolares}

\section{Comparison of three overweight and obesity criteria among preschoolers}

Milena Baptista Bueno1

Regina Mara Fisberg 1

1Departamento de Nutrição. Faculdade de Saúde Pública. Universidade de São Paulo. Av. Dr Arnaldo, 715. São Paulo, SP, Brasil. CEP: 01.246-904. E-mail: mibueno@usp.br

\footnotetext{
Abstract

Introduction: There is not a consensus about the diagnostic of overweight and obesity in children. The recommendations by World Health Organization (WHO), Center of Disease Control (CDC) and International Obesity Task Force (IOTF) are more commons. There are differences among the index (BMI or score), population (american or others) and/or conception.

Objectives: to compare three nutritional status classifications in a representative sample of preschoolers, whose attended regularly public daycare centers in São Paulo city.

Methods: Twenty-one day-care centers and 676 children, between 2 and 7 years old, were randomly assigned by a cluster sampling in two stages. Each child was classified about his nutritional status using three different criteria and the concordance among the indexes were analyzed by Kappa statistics. The prevalence of overweight was $18.6 \%, 13.2 \%$ and $12.2 \%$ according to the indexes WHO, CDC and IOTF, respectively.

Results: About the overweight, the range of prevalence difference was $2,7 \%$ until $12,4 \%$ and for obesity, the range was 3,7\% until 5,4\%. Highlighting the difference between indexes proposed by CDC and IOTF, mainly in males obesities, which the CDC was 2.6 higher. The concordance was low $(k<0,40)$ only between males overweight classified by $C D C$ and IOTF.

Conclusions: It is the possible to compare studies which use different criteria to classify nutritional status in children but they need to be done carefully.

Key words obesity, preschoolers, nutritional status index, day-care centers
}

\section{Resumo}

Introdução: Não há consenso sobre o critério diagnóstico para sobrepeso e obesidade infantil. Os mais utilizados são os recomendados pela Organização Mundial da Saúde (OMS), pelo Centers for Disease Control and Prevention (CDC) e pelo International Obesity Task Force (IOTF), diferenciando-se no índice (IMC ou escore-Z), população (americana ou agregando outras) elou concepção.

Objetivos: comparar os 3 critérios de classificação do estado nutricional em uma amostra representativa de pré-escolares matriculados em creches públicas de São Paulo.

Métodos: 21 creches públicas do município de São Paulo e 676 crianças de 2 a 7 anos foram sorteadas aleatoriamente por um processo de amostragem por conglomerados em duas etapas. Cada criança foi classificada segundo os 3 critérios e a concordância entre os indicadores foi avaliada pela estatística de Kappa. A prevalência de sobrepeso, classificada pelos critérios da OMS, CDC e IOTF, foi de 18,6\%, 13,2\% e 12,2\%, respectivamente.

Resultados: Verificou-se oscilação na diferença dos valores de prevalência de sobrepeso entre 2,7 a 12,4e obesidade entre 3,7 a 5,4 pontos percentuais. Destacouse o índice proposto pelo CDC quando comparado ao IOTF, principalmente entre obesos do sexo masculino, sendo o CDC até 2,6 maior. A concordância foi baixa $(k$ $<0,40$ ) somente entre sobrepesos do sexo masculino classificados segundo o CDC e IOTF.

Conclusão: a comparação entre os estudos que utilizam diferentes critérios de classificação do estado nutricional deve ser realizada cautelosamente.

Palavras-chave Obesidade, Pré-escolar, Indicadores do estado nutricional, Creche 


\section{Introdução}

Em alguns países da América Latina que apresentaram rápido crescimento econômico nas últimas décadas, observou-se tendência temporal de diminuição da desnutrição infantil associado ao aumento da prevalência da obesidade. ${ }^{1}$ No Brasil, esta transição nutricional ainda não é muito evidente, ${ }^{2}$ porém existem relatos de que a prevalência de obesidade supera a de desnutrição infantil. 3,4

$\mathrm{O}$ início da obesidade pode ocorrer ainda nas idades mais tênues do ser humano e persistir até a idade adulta. ${ }^{5}$ Assim, o diagnóstico precoce de sobrepeso ou obesidade é de grande interesse para a saúde pública, pois o tratamento nesta fase inicial da vida pode ser mais eficiente e menos oneroso ao Estado. 6

A obesidade está relacionada ao aumento do risco e surgimento de doenças, tais como as cardiovasculares. Na infância é difícil avaliar a obesidade devido à intensa modificação da estrutura corporal (massa óssea, massa magra, água e gordura) durante o crescimento. 7,8 Sendo assim, não existe um sistema de classificação de obesidade infantil universalmente aceito.

O diagnóstico de sobrepeso e obesidade em crianças recomendado pela Organização Mundial da Saúde (OMS) baseia-se na distribuição de escore-Z de peso para altura, que é a relação entre o peso encontrado e o peso ideal para a altura. ${ }^{9}$

Por um longo período, este índice foi o único utilizado para identificar desvios nutricionais na infância. Em 2000, o Centers for Disease Control and Prevention (CDC) publicou uma revisão das curvas recomendadas pelo NCHS a fim de corrigir ou minimizar falhas que o apontam como um indicador imperfeito, tais como: aprimoramento das técnicas estatísticas, ampliação das amostras, padronização da coleta de dados e exclusão de dados que comprometiam a população de referência. Entretanto, o fator de inovação que mais se destacou foi o desenvolvimento de um novo índice (Índice de Massa Corporal - IMC, por idade) com maior sensibilidade e especificidade quando comparado ao escore- $Z$ de peso para altura para detectar excesso de peso em crianças a partir de 2 anos. 10

Neste mesmo ano, Cole et al. ${ }^{11}$ divulgaram curvas de IMC (2-18 anos), a partir de dados obtidos de pesquisas populacionais de seis países, incluindo Brasil e Estados Unidos, conferindo-lhes um caráter internacional e por isso recomendadas pela International Obesity Task Force (IOTF). Um diferencial deste padrão de referência é que os pontos de corte para classificação de sobrepeso e obesidade por sexo e faixa etária foram definidos através da interceptação, aos 18 anos de idade, com os pontos de IMC já utilizados para classificação de sobrepeso $\left(25 \mathrm{a} 30 \mathrm{~kg} / \mathrm{m}^{2}\right)$ e obesidade $\left(>30 \mathrm{~kg} / \mathrm{m}^{2}\right)$ em adultos e, a partir desses valores, foram construídas curvas para as idades menores de 18 anos. Segundo os autores, os pontos de corte estabelecidos por este método são menos arbitrários, já que são baseados nos pontos de corte de IMC para adultos que melhor predizem morbidade e mortalidade.

Considerando que o uso dos diferentes métodos de classificação pode influenciar na estimativa de sobrepeso e obesidade infantil, julgou-se interessante comparar os 3 critérios de análise do estado nutricional infantil em uma amostra de pré-escolares matriculados em creches públicas do município de São Paulo.

\section{Métodos}

Este estudo é parte de um projeto temático denominado "Nutrição e Saúde: Uma Abordagem Integrada para Avaliação Nutricional, Desenvolvimento de Alimentos para Fins Especiais e Intervenção Nutricional", desenvolvido pelo Departamento de Nutrição da Faculdade de Saúde Pública da Universidade de São Paulo.

Os dados antropométricos avaliados foram obtidos na primeira coleta de um estudo longitudinal representativo das crianças que freqüentam creches públicas do município de São Paulo. Essa coleta ocorreu no mês de outubro do ano de 1999, quando foram visitadas 21 creches e avaliadas 676 crianças de 2 a 7 anos, sorteadas por um processo de amostragem por conglomerados em duas etapas. 4

Para o cálculo do tamanho da amostra considerou-se uma prevalência de $5 \%$ de desnutrição, valor aproximado entre crianças menores de 5 anos em famílias do menor estrado de renda do município. ${ }^{2}$ Neste mesmo estudo, a prevalência de obesidade (escore- $Z$ de peso para altura maior que 2 ) foi de $1,9 \%$ e não é citada a prevalência de sobrepesos, mas supõe-se que a soma das prevalências de obesos e sobrepesos seja próxima a de desnutrição.Assim, podemos supor que esse tamanho de amostra está muito próximo ao valor que seria obtido se utilizássemos a prevalência de excesso de peso infantil. Para o cálculo, admitiu-se como aceitável uma precisão tal que, em $95 \%$ das possíveis amostras, o erro máximo não ultrapassasse $1,5 \%$ e adicionaramse $5 \%$ do valor obtido devido às perdas estimadas.

Em 1999, o município de São Paulo atendia aproximadamente 80.000 crianças entre 4 e 83 
Classificação de sobrepeso e obesidade infantil.

\begin{tabular}{|lll|}
\hline Referência & Sobrepeso & Obesidade \\
\hline OMS & escore-Z de peso para altura entre 1 e 2 & escore-Z de peso para altura > 2 \\
CDC & IMC entre os percentis 85 e 95 & IMC acima do percentil 95 \\
IOTF & Ponto de corte equivalente ao IMC & Ponto de corte equivalente ao IMC \\
& adulto entre 25 e 30 & adulto maior do que 30 \\
\hline
\end{tabular}

meses, distribuídas em 718 unidades. O procedimento de amostragem foi por conglomerados em duas etapas. Para garantir a representatividade das regiões do município de São Paulo, o sistema de referência para a primeira etapa de amostragem foi ordenado segundo regiões administrativas. Caso a creche fosse de tamanho reduzido, eram agrupadas a fim de igualar a capacidade de atendimento. Utilizando-se um procedimento de amostragem sistemática, obteve-se uma amostra de 21 creches estratificadas por região. As crianças a serem avaliadas nas creches amostradas foram selecionadas por sorteio sistemático, depois de calculada a fração global da amostra.

Para a obtenção do peso corporal utilizou-se balança digital com capacidade para $150 \mathrm{~kg}$ e precisão de $200 \mathrm{~g}$. Somente uma peça íntima foi permitida na pesagem. Para a aferição da altura, as crianças foram medidas na posição em pé, descalças, com o auxílio de um estadiômetro vertical, a partir de um microestadiômetro da marca "Stanley" (precisão $0,1 \mathrm{~cm}$ ).

A padronização das medidas antropométricas para controle da variabilidade inter e intra entrevistadores seguiram as recomendações de Lohman et al. 12 e foi realizada no laboratório do Núcleo de Pesquisas Epidemiológicas em Nutrição e Saúde da Universidade de São Paulo. Os procedimentos técnicos para a realização das medidas antropométricas foram padronizados, utilizando-se os métodos propostos por Habicht et al. 13

A digitação foi realizada em duplicata a fim de comparar as digitações pelo módulo "VALIDATE" do programa Epi Info versão 6.04.14 Os campos não coincidentes foram conferidos nos originais e retificados. Os índices antropométricos recomendados para classificação da OMS e CDC foram calculados no módulo "EPINUT" do programa Epi Info versão 2000 para Windows. ${ }^{15}$

A prevalência de sobrepeso e obesidade entre pré-escolares que freqüentavam creches foi calculada utilizando os critérios apresentados no Quadro
1. As crianças foram divididas em duas faixas etárias (2 l-- 4 e 4 l-- 7 anos) a fim de visualizar o comportamento dos indicadores de excesso de peso nas diferentes fases de crescimento infantil.

Os resultados foram apresentados em proporções e intervalo de confiança. Foi calculada a diferença de prevalências de sobrepeso e obesidade entre os critérios e aplicado o teste de probabilidade binomial, adotando o nível de significância de 0,05. Para mensurar a concordância entre os critérios utilizouse a estatística Kappa $(k) .16$

A anuência para participar da pesquisa foi feita por escrito, com assinatura do termo de consentimento livre e esclarecido pelos responsáveis da criança. Este estudo está de acordo com os requisitos da Resolução do Conselho Nacional de Saúde 196/ 96 e foi aprovado pelo Comitê de Ética da Faculdade de Saúde Pública da Universidade de São Paulo.

\section{Resultados}

A média de idade das crianças foi de 4,12 anos (DP $=1,28$ anos). A distribuição das faixas etárias foi semelhante entre os sexos $(p>0,05)$, conforme mostra a Tabela 1.

Tabela 1

Distribuição de crianças frequentadoras de creches públicas segundo faixa etária e sexo.

\begin{tabular}{|c|c|c|c|c|c|c|}
\hline \multirow{3}{*}{$\begin{array}{l}\text { Faixa etária } \\
\text { (anos) }\end{array}$} & \multicolumn{4}{|c|}{ Sexo } & \multicolumn{2}{|c|}{ Total } \\
\hline & \multicolumn{2}{|c|}{ Masculino } & \multicolumn{2}{|c|}{ Feminino } & \multirow[b]{2}{*}{$\mathrm{n}$} & \multirow[b]{2}{*}{$\%$} \\
\hline & $\mathrm{n}$ & $\%$ & $\mathrm{n}$ & $\%$ & & \\
\hline $2 \mid--3$ & 73 & 21,9 & 85 & 24,8 & 158 & 23,4 \\
\hline $3 \mid--4$ & 92 & 27,6 & 90 & 26,2 & 182 & 26,9 \\
\hline $41--5$ & 84 & 25,2 & 68 & 19,8 & 152 & 22,5 \\
\hline 5 |- 6 & 54 & 16,3 & 59 & 17,2 & 113 & 16,7 \\
\hline $61--7$ & 30 & 9,0 & 41 & 11,9 & 71 & 10,5 \\
\hline Total & 333 & 100,0 & 343 & 100,0 & 676 & 100,0 \\
\hline
\end{tabular}

$p>0,05$ 
Tabela 2

\begin{tabular}{|c|c|c|c|c|c|c|c|}
\hline \multirow[t]{2}{*}{ Faixa etária (anos) } & \multirow[t]{2}{*}{ Sexo } & \multicolumn{3}{|c|}{$\begin{array}{l}\text { Sobrepeso } \\
\text { (IC 95\%) }\end{array}$} & \multicolumn{3}{|c|}{$\begin{array}{l}\text { Obesidade } \\
\text { (IC 95\%) }\end{array}$} \\
\hline & & OMS & $\mathrm{CDC}$ & IOTF & OMS & $\mathrm{CDC}$ & IOTF \\
\hline \multirow[t]{2}{*}{$2 \mathrm{l}-\mathrm{-} 4$ anos } & $\begin{array}{l}\text { Feminino } \\
(\mathrm{n}=175)\end{array}$ & $\begin{array}{c}18,7 \\
(12,7-24,7)\end{array}$ & $\begin{array}{c}13,7 \\
(8,6-18,9)\end{array}$ & $\begin{array}{c}13,2 \\
(8,0-18,3)\end{array}$ & $\begin{array}{c}5,1 \\
(1,8-8,4)\end{array}$ & $\begin{array}{c}8,6 \\
(4,4-12,8)\end{array}$ & $\begin{array}{c}4,6 \\
(1,4-7,7)\end{array}$ \\
\hline & $\begin{array}{c}\text { Masculino } \\
(n=165)\end{array}$ & $\begin{array}{c}19,2 \\
(13,1-25,4)\end{array}$ & $\begin{array}{c}12,7 \\
(7,6-17,9)\end{array}$ & $\begin{array}{c}6,8 \\
(2,9-10,8)\end{array}$ & $\begin{array}{c}2,4 \\
(0,05-4,8)\end{array}$ & $\begin{array}{c}6,1 \\
(2,4-9,7)\end{array}$ & $\begin{array}{c}2,4 \\
(0,05-4,8)\end{array}$ \\
\hline \multirow[t]{2}{*}{$4 \mathrm{l}-\mathrm{-}$ anos } & $\begin{array}{l}\text { Feminino } \\
(\mathrm{n}=168)\end{array}$ & $\begin{array}{c}19,9 \\
(13,4-26,3)\end{array}$ & $\begin{array}{c}14,9 \\
(9,4-20,3)\end{array}$ & $\begin{array}{c}17,2 \\
(11,2-23,2)\end{array}$ & $\begin{array}{c}10,1 \\
(5,5-14,7)\end{array}$ & $\begin{array}{c}10,1 \\
(5,5-14,7)\end{array}$ & $\begin{array}{c}6,6 \\
(2,8-10,3)\end{array}$ \\
\hline & $\begin{array}{c}\text { Masculino } \\
(n=168)\end{array}$ & $\begin{array}{c}16,7 \\
(10,7-22,6)\end{array}$ & $\begin{array}{c}11,3 \\
(6,5-16,1)\end{array}$ & $\begin{array}{c}11,9 \\
(6,8-16,9)\end{array}$ & $\begin{array}{c}7,1 \\
(3,2-11,1)\end{array}$ & $\begin{array}{c}12,5 \\
(7,4-17,5)\end{array}$ & $\begin{array}{c}4,8 \\
(1,5-8,0)\end{array}$ \\
\hline Total & & $\begin{array}{c}18,6 \\
(15,6-21,6)\end{array}$ & $\begin{array}{c}13,2 \\
(10,6-15,7)\end{array}$ & $\begin{array}{c}12,2 \\
(9,7-14,8)\end{array}$ & $\begin{array}{c}6,2 \\
(4,4-8,0)\end{array}$ & $\begin{array}{c}9,3 \\
(7,1-11,5)\end{array}$ & $\begin{array}{c}4,6 \\
(3,0-6,2)\end{array}$ \\
\hline
\end{tabular}

Gráfico 1

Diferença de prevalências de sobrepeso quando adotado o critério da Organização Mundial da Saúde (OMS) e os propostos pelo Centers for Disease Control and Prevention (CDC) e International Obesity Task Force (IOTF), segundo sexo e faixa etária.

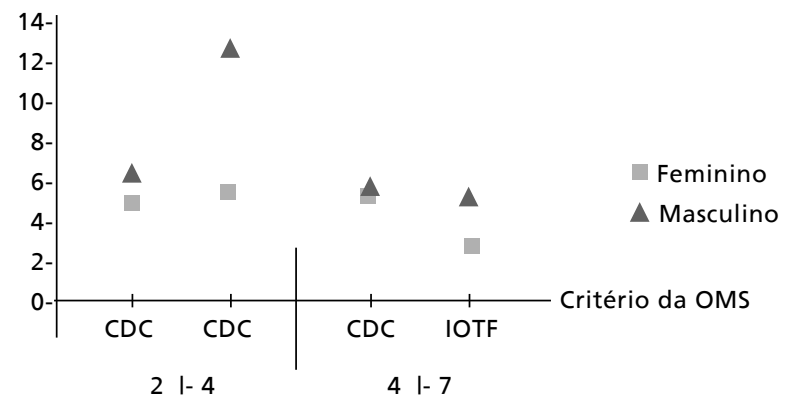

A prevalência de sobrepeso segundo os critérios OMS, CDC e IOTF está apresentada na Tabela 2. Essa prevalência foi maior para qualquer sexo e faixa etária quando adotado o critério da OMS; a diferença de freqüência de sobrepeso adotando esse critério e os restantes (CDC e IOTF) variou, de $2,7 \%$ a $12,4 \%$ (Gráfico 1). Destacou-se a diferença entre prevalências de sobrepeso no sexo masculino, principalmente na faixa etária de 2 a 4 anos, no qual a prevalência calculada pelo padrão OMS foi 2,8 vezes maior do que a calculada pelo padrão IOTF ( $\mathrm{p}$ $<0,05)$ e 1,5 vezes maior quando adotado o padrão CDC $(p<0,05)$.

Entre os critérios CDC e IOTF, a diferença da prevalência de sobrepeso entre crianças de 2 a 4 anos foi de $0,5 \%(p>0,05)$ e $5,9 \%(p<0,05)$, entre o sexo feminino e masculino respectivamente. Entre as crianças maiores (4 a 7 anos) a diferença foi de $2,3 \%(p>0,05)$ entre meninas e $0,6 \%(p>0,05)$ entre meninos, porém, a prevalência calculada pelo critério adotado pelo IOTF foi superior, ao contrário do que ocorreu entre as crianças com idades entre 2 e 4 anos.

Em relação à obesidade, quando estratificada a amostra por sexo e idade, verificou-se oscilação da diferença de prevalências de $-3,7 \%$ a $5,4 \%$ entre o critério adotado pela OMS e os restantes (Gráfico 2). Destacou-se o índice proposto pelo CDC quando comparado com os outros dois critérios entre as crianças de sexo masculino das duas faixas etárias ( $\mathrm{p}$ $<0,05)$, sendo até 2,6 vezes maior.

A Tabela 3 mostra a concordância entre os critérios avaliada pela estatística Kappa, apresentando valores de 0,22 a 1,00 . Nota-se que a concordância foi mais fraca entre sobrepesos do sexo masculino classificados segundo IMC utilizando os critérios do CDC e do IOTF. 
Coeficiente Kappa (IC 95\%) entre os critérios de classificação do estado nutricional, segundo sexo e faixa etária.

\begin{tabular}{|c|c|c|c|c|c|c|c|}
\hline \multirow[t]{3}{*}{ Estado Nutricional } & \multirow[t]{3}{*}{ Sexo } & \multicolumn{6}{|c|}{ Faixa etária (anos) } \\
\hline & & \multicolumn{3}{|c|}{$21--4$} & \multicolumn{3}{|c|}{4 ।-- 7} \\
\hline & & OMS e CDC & OMS e IOTF & CDC e IOTF & OMS e CDC & OMS e IOTF & CDC e IOTF \\
\hline \multirow[t]{2}{*}{ Sobrepeso } & Masculino & $\begin{array}{c}0,68 \\
(0,53-0,83)\end{array}$ & $\begin{array}{c}0,47 \\
(0,34-0,60)\end{array}$ & $\begin{array}{c}0,24 \\
(0,10-0,39)\end{array}$ & $\begin{array}{c}0,53 \\
(0,37-0,68)\end{array}$ & $\begin{array}{c}0,77 \\
(0,62-0,92)\end{array}$ & $\begin{array}{c}0,22 \\
(0,07-0,38)\end{array}$ \\
\hline & Feminino & $\begin{array}{c}0,8 \\
(0,65-0,95)\end{array}$ & $\begin{array}{c}0,77 \\
(0,62-0,91)\end{array}$ & $\begin{array}{c}0,6 \\
(0,44-0,75)\end{array}$ & $\begin{array}{c}0,7 \\
(0,55-0,86)\end{array}$ & $\begin{array}{c}0,98 \\
(0,81-1,14)\end{array}$ & $\begin{array}{c}0,77 \\
(0,61-0,92)\end{array}$ \\
\hline \multirow[t]{2}{*}{ Obesidade } & Masculino & $\begin{array}{c}0,56 \\
(0,42-0,69)\end{array}$ & $\begin{array}{c}1 \\
(0,85-1,15)\end{array}$ & $\begin{array}{c}0,56 \\
(0,42-0,71)\end{array}$ & $\begin{array}{c}0,43 \\
(0,29-0,58)\end{array}$ & $\begin{array}{c}0,57 \\
(0,43-0,72)\end{array}$ & $\begin{array}{c}0,52 \\
(0,38-0,65)\end{array}$ \\
\hline & Feminino & $\begin{array}{c}0,73 \\
(0,59-0,87)\end{array}$ & $\begin{array}{c}0,81 \\
(0,66-0,97)\end{array}$ & $\begin{array}{c}0,68 \\
(0,54-0,82)\end{array}$ & $\begin{array}{c}0,34 \\
(0,19-0,50)\end{array}$ & $\begin{array}{c}0,46 \\
(0,31-0,61)\end{array}$ & $\begin{array}{c}0,77 \\
(0,62-0,91)\end{array}$ \\
\hline
\end{tabular}

\section{Discussão}

A prevalência de sobrepeso foi maior para qualquer faixa etária e sexo quando utilizado o critério da OMS, ou seja, a utilização de qualquer um dos outros dois critérios subestimaria a prevalência de sobrepeso, especialmente o critério recomendado pelo IOTF.

Apesar de provenientes de pesquisas populacionais norte-americanas, os critérios do CDC e OMS não apresentaram resultados idênticos. A idade é a principal variável que diferencia os critérios recomendados pela OMS e CDC, pois o peso para altura, adotado pela OMS, estabelece que o peso aumenta com a altura, independente da idade e, o IMC é estabelecido em função do tempo de vida da criança. O IMC reflete melhor a gordura corporal, principalmente em crianças maiores de 5 anos. A adoção do IMC para análise do estado nutricional infantil tende a ser recomendada como padrão internacional de referência por todos os institutos, porém esta transição terá implicações nas comparações com estudos anteriores que utilizavam o padrão peso para altura, recomendado por mais de vinte anos. 17

A maior variação da prevalência de sobrepeso e obesidade classificados segundo OMS e CDC foi de 6,5 e 5,4 pontos percentuais, respectivamente. Flegal et al.18 observaram amplitude de variação da prevalência de sobrepeso semelhante em crianças americanas com 4 anos de idade, no entanto, nas outras faixas etárias (2, 3 e 5 anos) a amplitude foi menor, em torno de $1,5 \%$. Kain et al. ${ }^{19}$, estudando crianças chilenas com 6 anos de idade, verificaram
Gráfico 2

Diferença de prevalências de obesidade quando adotado o critério da Organização Mundial da Saúde (OMS) e os propostos pelo Centers for Disease Control and Prevention (CDC) e International Obesity Task Force (IOTF), segundo sexo e faixa etária

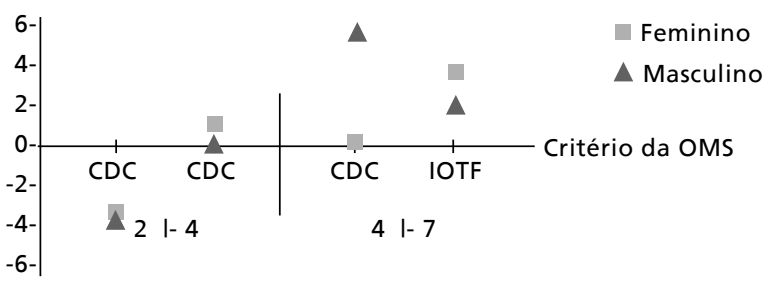

diferenças menores entre esses dois critérios $(0,8 \%$ a $3,3 \%)$.

Flegal et al.,20 analisando os dados de crianças de 2 a 5 anos dos inquéritos nacionais americanos NHANES I, II e III, observaram que a diferença entre as prevalências de sobrepeso de meninos classificados segundo os padrões recomendados pelo IOTF e CDC variou de 5,6 a 9 pontos percentuais. Entre as meninas a faixa de variação foi menor, entre 3,5 a $4,3 \%$. Esses valores concordam com os do presente estudo em relação ao sexo masculino $(5,9 \%)$, mas se distanciam ao compararmos com o do sexo feminino $(0,5 \%)$.

Existem estudos que propõem a criação de 
padrão de referência utilizando o IMC em populações especificas, inclusive brasileira.21-25 Esses novos padrões mostram a realidade da região de abrangência, porém dificultam a comparação entre estudos de diferentes regiões. A proposta de Cole $e t$ $a l .11$ minimiza estes erros já que possui caráter internacional. No entanto, a utilização desse padrão não é consensual. Reilly et al. ${ }^{26}$ sugerem que o padrão adotado pelo IOTF tem baixa sensibilidade para obesidade, pois em outros estudos, 19,20,26 esse critério apresentou menor prevalência de excesso de peso infantil, principalmente, obesidade.

Sotelo et al. 27 observaram que a obesidade classificada segundo o critério recomendado pela IOTF entre escolares foi menor que a prevalência dessa patologia quando classificada pela OMS. O mesmo não ocorreu com o sobrepeso, que apresentou prevalências próximas entre os critérios adotados, principalmente entre o sexo feminino. Comparando com os pré-escolares analisados neste estudo, o comportamento dos indicadores de obesidade e sobrepeso foi semelhante, no entanto, entre os com idade entre 2 e 4 anos do sexo masculino, a diferença de valores da prevalência de sobrepeso foi mais evidente.

Os critérios de classificação de excesso de peso infantil diferenciam-se segundo índice (IMC ou escore-Z), população (americana ou agregando outras) e/ou concepção, portanto diferenças entre as prevalências eram esperadas, independentes de sua magnitude. Não há um padrão da diferença entre os índices segundo sexo e faixa etária, ou seja, não há um erro sistemático, como já identificado em outros estudos, 19,20,26 dificultando a compreensão das justi-

\section{Referências}

1. Martorell R, Khan LK, Hughes ML, Grummer-Strawn LM. Overweight and obesity in preschool children from developing countries. Int J Obes 2000; 24:959-67.

2. Monteiro CA, Conde WL. Tendência secular da desnutrição e obesidade na infância na cidade de São Paulo (19741996). Rev Saúde Pública 2000; 34 (6 Suppl): 52-61.

3. Balaban G, Silva GAP. Prevalência de sobrepeso e obesidade em crianças e adolescentes de uma escola da rede privada de Recife. J Pediatr (Rio de J) 2001; 77: 96-100.

4. Bueno MB, Marchioni DML, Fisberg RM. Evolução nutricional de crianças atendidas em creches públicas no município de São Paulo. Rev Panam Salud Pública 2003; 14: $165-8$.

5. Guo SS, Roche AF, Chumlea WC, Gardner JD, Siervogel RM. The predictive value of childhood body mass index values for overweight at age 35 year. Am J Clin Nutr 1994; 59: 810-9. ficativas dessas diferenças e implicações na comparação de estudos.

Independente das diferenças percentuais, a concordância entre a maioria dos critérios apresentou-se como boa $(k>0,40)$. A exceção foi verificada entre sobrepesos do sexo masculino classificados segundo os critérios propostos pelos IOTF e CDC. Abrantes et al. ${ }^{28}$ mostraram que os índices propostos pelo IOTF e OMS têm boa concordância entre pré-escolares, principalmente entre sobrepesos, corroborando com os observados no presente estudo. Assim, a comparação entre estudos que utilizam diferentes índices pode ser realizada, porém com cautela para alguns grupos de pré-escolares.

A falta do "padrão-ouro" para classificação do estado nutricional infantil na população com medidas antropométricas de fácil obtenção e baixo custo, como peso e altura, implica no comprometimento do planejamento e ações de saúde pública em obesidade infantil, tema cada vez mais relevante na sociedade contemporânea. As diferenças percentuais entre os critérios de classificação podem corresponder a uma grande quantidade de crianças. $\mathrm{O}$ conhecimento da diferença da classificação do estado nutricional entre os critérios mais utilizados no meio científico e nos serviços de saúde, torna mais evidentes os erros ao comparar populações que adotaram distintos critérios.

\section{Agradecimentos}

Pesquisa financiada pela Fundação de Amparo a Pesquisa do Estado de São Paulo (FAPESP), processo 98/08095-9.
6. Neovius M, Linné $\mathrm{Y}$, Barkeling $\mathrm{B}$, Rossner $\mathrm{S}$. Discrepancies between classification systems of childhood obesity. Obes Rev 2004; 5:105-14.

7. Daniels SR, Morrison JA, Sprecher DL, Khoury P, Kimball TR. Association of body fat distribution and cardiovascular risk factors in children and adolescents. Circulation 1999; 99: 541-5.

8. Teixeira PJ, Sardinha LB, Going SB, Lohman TG. Total and regional fat and serum cardiovascular disease risk factors in lean and obese children and adolescents. Obes Res 2001; 9: 432-42.

9. World Health Organization. The use and interpretation of antropometry. Geneva: WHO; 1995.

10. Kuczmarski RJ, Ogden CL, Grummer-Strawn LM, Flegal KM, Guo SS, Wei R, et al. CDC growth charts: United States. Adv Data 2000; (314): 1-27. 
11. Cole TJ, Bellizzi MC, Flegal KM, Dietz WH. Estabilishing a standard definition for child overweight and obesity worldwide: international survey. BMJ 2000; 320: 1240-3.

12. Lohman TG, Roche AF, Martorell R. Anthropometric standardization reference manual. Champaign: Human Kinetics Books; 1988.

13. Habicht JP, Martorell R, Yarbrough C, Malina RM, Klein RE. Height and weight standard for preschool children: how relevante are ethnic differences in growth potential? Lancet 1974; 1: 611-5.

14. Dean AG, Dean JA, Coulombier D, Brendel KA, Smith DC, Burton HA, et al. Epi Info, version 6.04: a word processing database and statistics program for a epidemiology on microcomputers. Atlanta: Centers for Disease Control and Prevention; 1996.

15. Dean AG, Arner TG, Sangan S, Sunki GG, Friedman R, Lantinga M et al. Epi Info 2000, a database and statistics program for public health professionals for use on Windows 94, 98, NT and 2000 computers. Atlanta: Center for Disease Control and Prevention; 2000.

16. Dawson B, Trapp RG. Basic \& clinical biostatistics. New York: McGraw-Hill; 2001.

17. Soares NT. Um novo referencial antropométrico de crescimento: significados e implicações. Rev Nutr 2003; 16: 93 104.

18. Flegal KM, Wei R, Ogden C. Weight for stature compared with body mass index for age growth charts for the United States from the Centers for Disease Control and Prevention. Am J Clin Nutr 2002; 75: 761-6.

19. Kain J, Uauy R, Vio F, Allbala C. Trends in overweight and obesity prevalence in Chilean children: comparison of three definitions. Eur J Clin Nutr 2002; 56: 200-4.

20. Flegal KM, Ogden CL, Wei R, Kuczmarski RL, Jonhnson CL. Prevalence of overweight in US children: comparison of US growth charts from the Centers for Disease Control and Prevention with other reference values for body mass index. Am J Clin Nutr 2001; 73: 1086-93.
21. Conde WL. Desenvolvimento e aplicação de sistema classificatório para avaliação do estado nutricional de crianças e adolescentes brasileiros baseado no índice de massa corporal [Tese de Doutorado]. São Paulo: Departamento de Nutrição, Faculdade de Saúde Pública da Universidade de São Paulo; 2004.

22. Fredricks AM, Buuren SV, Burgmeijer RJF, Meulmeester JF, Beuker RJ, Brugman E, et al. Continuing positive secular growth change in the Nevertherlands 1995-1997. Pediatr Res 2000; 47: 316-23.

23. Schaefer F, Georgi M, Wül E, Schärer K. Body mass index and percentage fat mass in healthy German schoolchildren and adolescents. Int J Obes 1998; 24: 1623-7.

24. Luciano A, Bressan F, Zoppi G. Body mass index reference for children aged 3 - 19 years from Verona, Italy. Eur J Clin Nutr 1997; 51: 6-10.

25. White EM, Wilson AC, Greene AS, McCowan CC, Thomas GE, Cairns AY, et al. Body mass index centile charts to assess fatness of British children. Arch Dis Child 1995; 72 : 38-41.

26. Reilly JJ, Dorosty AR, Emmett PM. Identification of the obese child: adequacy of the body mass index for clinical practice and epidemiology. Int J Obes Relat Metab Disord 2000; 24: 1623-7.

27. Sotelo YOM, Colugnati FAB, Taddei JAAC. Prevalência de sobrepeso e obesidade entre escolares da rede pública segundo três critérios de diagnóstico antropométrico. Cad Saúde Pública 2004; 20: 233-40.

28. Abrantes MM, Lamounier JA, Colosimo EA. Comparison of body mass index values proposed by Cole et al. (2000) and Must et al. (1991) for identifying obese children with weight-for-height index recommended by the World Health Organization. Public Health Nutr 2003; 6: 307-11.

Recebido em 27 de abril de 2005

Versão final apresentada em 13 de novembro de 2006.

Aprovado em 30 de novembro de 2006 